\title{
Regurgitation Quantification Using 3D PISA in Volume Echocardiography
}

\author{
Leo Grady ${ }^{1}$, Saurabh Datta ${ }^{2}$, Oliver Kutter ${ }^{1}$, Christophe Duong ${ }^{2}$, \\ Wolfgang Wein ${ }^{3}$, Stephen H. Little ${ }^{4}$, Stephen R. Igo ${ }^{4}$, \\ Shizhen $\mathrm{Liu}^{5}$, and Mani Vannan ${ }^{5}$ \\ 1 Siemens Corporate Research, Princeton, NJ, USA \\ 2 Siemens Healthcare, Ultrasound Business Unit, Mountain View, CA, USA \\ 3 White Lion Technologies AG, Munich, Germany \\ 4 Methodist DeBakey Heart and Vascular Center, Houston, TX, USA \\ ${ }^{5}$ Ohio State University, Columbus OH, USA
}

\begin{abstract}
We present the first system for measurement of proximal isovelocity surface area (PISA) on a 3D ultrasound acquisition using modified ultrasound hardware, volumetric image segmentation and a simple efficient workflow. Accurate measurement of the PISA in 3D flow through a valve is an emerging method for quantitatively assessing cardiac valve regurgitation and function. Current state of the art protocols for assessing regurgitant flow require laborious and time consuming user interaction with the data, where a precise execution is crucial for an accurate diagnosis. We propose a new improved 3D PISA workflow that is initialized interactively with two points, followed by fully automatic segmentation of the valve annulus and isovelocity surface area computation. Our system is first validated against several in vitro phantoms to verify the calculations of surface area, orifice area and regurgitant flow. Finally, we use our system to compare orifice area calculations obtained from in vivo patient imaging measurements to an independent measurement and then use our system to successfully classify patients into mild-moderate regurgitation and moderate-severe regurgitation categories.
\end{abstract}

\section{Introduction}

Valvular heart diseases are a major cause of death in developed countries, which are estimated to affect $2.5 \%$ of the population and are the underlying cause of over 43700 deaths and 93000 hospital discharges in 2006 in the US. Pooled data from multiple studies suggest that the prevalence increases with age from $0.7 \%$ in participants $18-44$ years of age to $13.3 \%$ in participants $\geq 75$ years of age. The cause of these diseases can be congenital or acquired and, depending on the severity, treatment might involve medication, surgical valve repair or replacement. Timely diagnosis and accurate assessment of regurgitation severity is important for appropriate clinical decision making and optimal patient outcome [3].

Doppler ultrasound is routinely used to non-invasively evaluate intracardiac blood flow and detect regurgitant lesions for all cardiac valves. Several indexes

G. Fichtinger, A. Martel, and T. Peters (Eds.): MICCAI 2011, Part III, LNCS 6893, pp. 512-519, 2011.

(C) Springer-Verlag Berlin Heidelberg 2011 
have been developed to assess severity of regurgitation using color Doppler, Pulsed Wave (PW) and Continuous Wave (CW) Doppler. However, the use of color Doppler is limited to a semi-quantitative assessment of regurgitant jets directly or indirectly and is often dependent on user expertise. One direct, but laborious, method for quantifying regurgitation is the measurement of vena contracta $(\mathrm{VC})$, which is the narrowest cross-sectional area of the jet. Although $\mathrm{VC}$ measurement is a close approximation of the regurgitant orifice, this measure has limited accuracy due to dependence on system resolution, gain setting, acquisition view and user expertise [1].

The proximal isovelocity surface area (PISA) is a promising method for quantifying valve regurgitation. The PISA measurement is derived from the fluid dynamics principle that blood velocity increases as it approaches the valve orifice, forming concentric hemispheric shells of increasing velocity and decreasing surface area. Using appropriate settings of color Doppler imaging, these hemispheres can be visualized. Clinically, this estimation requires geometric assumptions and multiple steps. The effective regurgitant orifice area (EROA) or instantaneous flow rate $(\mathrm{mL} / \mathrm{s})$ can be computed from PISA via

$$
\mathrm{EROA}=\frac{\text { PISA } \times \text { Aliasing Velocity }}{\text { Peak Velocity }}
$$

The EROA is an important quantity to determine the best course of patient treatment [12]. Additionally, a PISA measurement makes it possible to directly calculate the regurgitation volume via

$$
\text { Regurgitation volume }=\int \mathrm{PISA} \times \text { Aliasing Velocity } d t .
$$

In 2D ultrasound acquisitions, the EROA is computed with a hemispheric geometric assumptions. Recently, 3D volume color Doppler acquisitions have made it possible to utilize more appropriate geometric assumptions [711] and provide better accuracy. However, these studies required a prolonged workflow involving multiple steps and computations, creating a barrier for clinical acceptance. Additionally, the volumetric color flow quantification accuracy in these studies is still dependent on system parameters such as acquisition (e.g., gated, stitched), angle, temporal and spatial resolution, system settings and workflow [5]. The clinical acceptance of the PISA technique will require real-time volume color Doppler capability on the acquisition system, an enhanced workflow, accurate quantification and elimination of geometric approximation. Fig. 1 illustrates the PISA and VC calculations from a color Doppler acquisition.

In this work, we use the capabilities of a state of the art real-time 3D cardiac ultrasound system in conjunction with $3 \mathrm{D}$ image segmentation techniques in order to improve the accuracy of the PISA measurement and simplify the workflow. Section 2 first explains our volumetric image acquisition hardware, and the segmentation algorithm used to extract the mitral annulus and the PISA surface. Validation on a range of in vitro and in vivo acquisitions are presented in Section 3. followed by a discussion in Section 4 . 

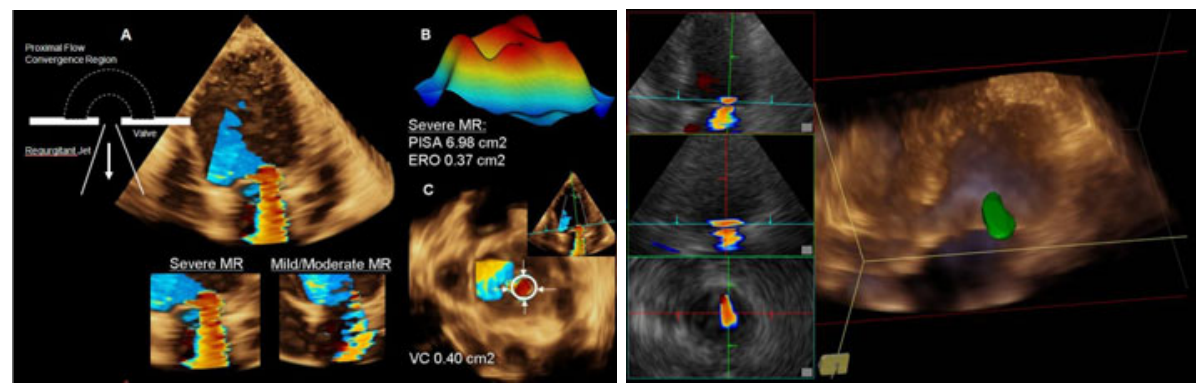

Fig. 1. Left: (A) Illustration of different cardiac mitral regurgitation (MR) and proximal isovelocity surface area (PISA) methods, (B) PISA surface generated from 3D manual measurement, (C) Manual measurement of vena contracta (VC). Right: An example PISA surface obtained from our system (different dataset).

\section{Methods}

Our system for accurate PISA quantification required hardware capability to acquire full volume color Doppler data along with software improvements. The hardware allows for sufficiently high volume rate acquisition and the software improvements were necessary to achieve an accurate and robust quantification. Our system was interactive, asking the user to place a point at the valve annulus and at the coaptation point (in the apical 4 or 2 chamber view).

\subsection{Echocardiography Imaging and Visualization}

We used a 2D matrix phased array ultrasound system (the ACUSON SC2000 ${ }^{\mathrm{TM}}$, 4Z1c transducer, which is now commercially available) for dual B-mode and color Doppler volume imaging. High volume rate acquisition is critical for an accurate quantification of the PISA surface volume, because low acquisition rates cause velocity discrepancies between multiple acquisition planes. These discrepancies create an inaccurate velocity surface estimation. The volume acquisition at higher rates is achieved by decoupling the transmit foci in the azimuthal and elevational directions. In azimuth, a plane-wave was used and in elevation, a virtual point source was used. For adequate spatial sampling of the volume in receive mode, we used a large number of parallel receive lines (40 or 64) for every transmit line. By combining a weakly focused transmit with many parallel receive lines, a high frame rate was achieved without a loss of spatial resolution. For example, a volume rate of $\approx 30 / \mathrm{s}$ is possible with fields of view of $70 \times 70^{\circ}$ in B-mode and $30 \times 30^{\circ}$ in color Doppler. Such volume rates are both comparable to conventional 2D color Doppler frame rates, and adequate for accurate PISA estimation. The exact imaging resolution depends on depth, but it was $\approx 1 \mathrm{~mm}$ for the Doppler acquisition and sub-mm for the B-mode acquisition. The imaging results in Fig. 1 were acquired using our system. 


\subsection{Localization of the Valve Annulus}

The extent of the valve annulus must be known in order to both quantify the flow through the valve and to provide a reference location from which to calculate the PISA surface. Our method can be used to localize the valve annulus for any target valve which has been specified by a single point inside the valve annulus (which we call the valve point).

Segmentation of the valve annulus is a challenging problem, since the valve is essentially a hole joining two chambers, creating poorly-defined boundaries. Additionally, the shape of the annulus and the surrounding anatomy varies depending on the target valve, patient and cardiac phase. We selected the random walker algorithm [4] for this segmentation task since it is known to behave well with poorly defined (or absent) boundaries and it makes no explicit assumptions on the annulus shape (which helps make the segmentation robust to large variability). Our system performs an interactive segmentation of the annulus, in contrast to [9] which employs an automatic, but more complex system.

Our strategy for utilizing the random walker algorithm for annulus segmentation is to automatically identify some voxels inside/outside the annulus which can be used to constrain the segmentation algorithm. In order to set seeds for the random walker algorithm, we adopt a simple model of the annulus (assuming an open valve) as a hypoechoic connection between two hypoechoic chambers. In order to determine the appropriate inputs to the random walker, we first determine the model orientation and then establish appropriate seeds. To improve the speed of the calculations, the annulus segmentation was limited to a $5 \mathrm{~cm} 3 \mathrm{D}$ ROI surrounding the valve point. For purposes of the model, we define a voxel to be hypoechoic if it falls below the median intensity in the ROI.

The orientation vector of the annulus model is determined by calculating the largest principal component of the hypoechoic voxels. Given the valve point and the orientation, we may identify annulus voxels (foreground seeds) for the random walker inside the annulus plane. The annulus plane is determined to be the hypoechoic part of the orthogonal plane connected to the valve point. In order to restrict the annulus segmentation, background seeds are placed parallel to the annulus plane on both sides at a distance of $2.5 \mathrm{~cm}$ (this value is used only to approximately set seeds and therefore does not need to be precise). With these input seeds, the random walker segmentation algorithm is employed to produce the annulus segmentation on the B-mode image. This approach to valve annulus segmentation may be applied to any of the cardiac valves (given a valve point), although PISA measurements are typically used to assess regurgitation at the mitral valve and left ventricular outflow tract (LVOT).

\subsection{PISA Measurement}

Once the location of the jet and valve coaptation point is located by the user, the PISA calculation may be performed. The identification of PISA in the 3D space allows for the computation of regurgitant flow during the cardiac phase of interest using (2). Our system expects that the desired isovelocity value and the 
direction of interest (relative to the annulus or valve coaptation line) is specified by the user and that the target isovelocity surface is contained entirely within the acquisition. Our strategy for PISA measurement is to generate a segmentation of the isovelocity region in the color Doppler data from which a surface can be segmented and a surface area may be calculated. Since the PISA is calculated above the coaptation point (relative to the valve annulus), the flows below the coaptation point (in the direction of the annulus) are ignored.

The isovelocity region above the coaptation point (i.e., in the direction away from the annulus) can be challenging to segment as a result of flow aliasing or confounding flow through a second valve (e.g., measuring PISA at the mitral valve may be confounded by the nearby flow through the LVOT). To account for these two challenges, we again use the random walker but this time we employ a novel directed graph which is designed to prevent cojoinment with a secondary flow. Specifically, for a target isovelocity value of $\gamma$, we establish the directed graph weight between two voxels $v_{i}$ and $v_{j}$ as $w_{i j}=1$ if $f_{i}, f_{j}>\gamma$ and $f_{i}<f_{j}$, or if $f_{i}, f_{j} \leq \gamma$, where $f_{i}$ represents the magnitude of the flow velocity at voxel $v_{i}$. Otherwise, $w_{i j}=\epsilon$. Optimization of the random walker with a directed graph is detailed in [10]. The coaptation point is treated as a foreground seed and the volume borders are treated as background seeds in the PISA segmentation.

The voxel-based segmentation result is smoothed using a 3D Gaussian kernel, and an isosurface mesh is successively computed using the Marching Cubes algorithm. The intersection with the mitral annulus segmentation is then removed from the mesh, yielding the hemispherical isosurface. Finally, all mesh vertex locations are transformed from acoustic to Cartesian space for computing the actual 3D PISA surface area measurement.

\section{$3 \quad$ Experiments and Results}

Our validation was designed to address the following issues: 1) Accuracy of the surface area computation, 2) Accuracy of the EROA calculation for various orifice shapes, relative to ground truth and relative to standard EROA approximation methods, 3) Accuracy of the regurgitant volume estimation, relative to ground truth and standard approximation methods, 4) Accuracy of EROA calculation on clinical patients, 5) The ability of our system to distinguish patients with mild-moderate regurgitation from patients with moderate-severe regurgitation. The first three issues were evaluated using a precise in vitro phantom (shown in Fig. 2) which could be controlled to produce calibrated flows and regurgitations through synthetic orifices of different shapes. The last two issues were probed using a retrospective study on a clinical patient population.

PISA segmentation and surface area computation was evaluated with a calibrated phantom. A hollow sphere with known surface area was imaged with the full volume 3D ultrasound system in the clinical configuration (shown in Fig. 2). The purpose of this experiment was to test the resolution limit and geometric consistency of the acquisition as a function of depth and beam overlap. Compared to the reference surface area of $49.09 \mathrm{~cm}^{2}$, the mean surface area computed by our system was $49.87 \mathrm{~cm}^{2}$ over five trials of acquisition/segmentation. 


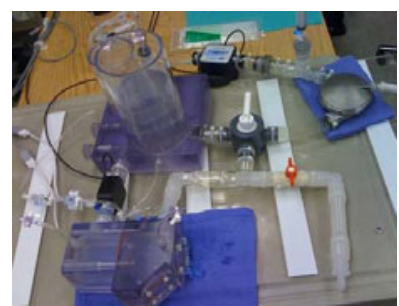

(a) In vitro validation setup

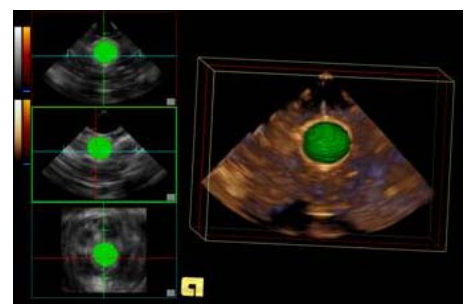

(b) Sphere validation

Fig. 2. Surface area validation. (a) Calibrated in vitro phantom. (b) Validation of our PISA quantification system for measuring the surface area of a sphere with known diameter.

To probe the accuracy of the EROA measurement, we created an in vitro phantom (described in [6]) with several orifice shapes in which the flow could be controlled to lie within a physiologically relevant severity of regurgitation (12$57 \mathrm{~mL} /$ beat). The flow loop was constructed to create physiologically relevant pressure differential and flow across the orifice of a regurgitant valve [6]. Data acquisition was performed using a standard clinical 3D full volume ultrasound scanner. Our method for measuring PISA was used to estimate the physiological flow convergence orifice and multiple frames were integrated to compute the flow for the entire cycle. A flow meter was placed in line to measure the true flow in the closed loop to measure regurgitation volume in the regurgitation flow loop. The values of effective regurgitant orifice area (EROA) and regurgitant volume were computed from the PISA measurements for 60 trials. The EROA measurement was compared with the true anatomic orifice area and the regurgitant volume was compared with the flow meter values for 32 trials. Additionally, we compared the same EROA measurements to the common clinical practice of hemispheric PISA estimation. Our system for PISA quantification consistently produced better EROA estimates than the reference method, as shown in Fig. 3. Note that the reference line in Fig. 3 refers to the value of the true orifice area. However, the physiologic orifice measured by the EROA is known to be $80-90 \%$ smaller than the true orifice area 2. We also computed the regurgitant volume from our system and compared this volume by the in line flow meter measurement used as the ground truth for regurgitant volume in our system (shown in Fig. 3). The conventional hemispheric method severely underestimated the EROA in all orifices and flow conditions including the circular orifice cases as shown in Fig. 3. Our method for measuring PISA performed better for all orifice shapes and flow conditions. The computation of regurgitant flow volumes also reflect a severe underestimation by the conventional PISA method when compared with the true regurgitant volume measured by a flow meter. In contrast, our PISA quantification system performed better in all cases irrespective of orifice geometry or flow condition as can be observed in Fig. 3. The in vitro work demonstrates the improvement in estimation of regurgitation using our method for measuring PISA in a controlled experimental setting. 


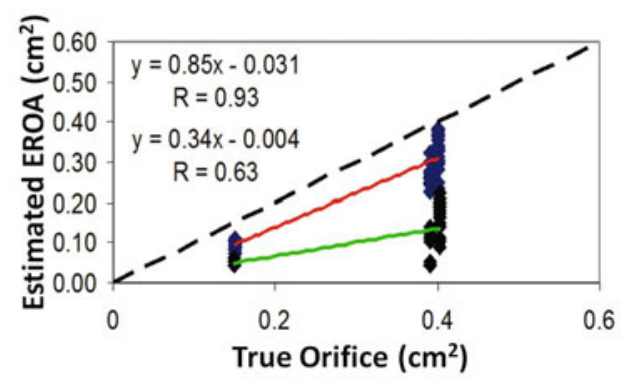

(a) $3 \mathrm{D}$ EROA

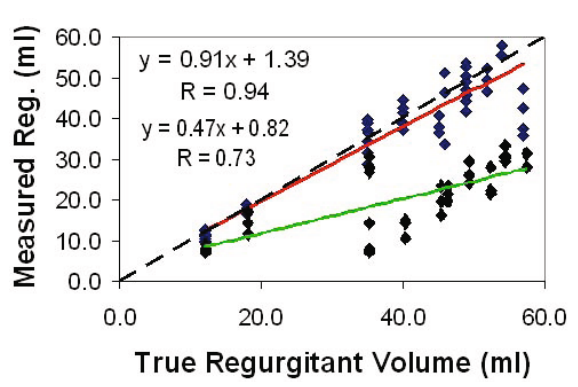

(b) 3D Pisa

Fig. 3. Results of experimental validation using an in vitro phantom to measure EROA and regurgitation volume. Bottom/green: Conventional spherical estimate. Top/red: System estimate. The spherical method severely underestimated the EROA in all orifices and flow conditions including the circular orifice (similar observations were made in 8]). Our method for measuring PISA performed better for all orifice shapes and flow conditions. The line in Fig. 3 refers to the value of the true orifice area, although the physiologic orifice measured by the EROA is known to be $80-90 \%$ smaller than the true orifice area [2].

In order to determine the accuracy of our system on patient data, we compared the EROA measurements obtained by our system with the measurement of vena contracta. Vena contracta measurements were performed manually by a clinical expert by tracing the flow convergence in a carefully positioned and aligned plane. The expert vena contracta measurements were compared with the EROA measurements obtained from our system. For a patient sample size of 33, we observed a statistically significant correlation $(\mathrm{R}=0.61, \mathrm{p}=0.002)$ between the EROA measurement and vena contracta measurements.

As a final test of our system on a clinical patient sample, we retrospectively examined a group of 35 patients which were segregated according to the clinical diagnosis of mitral regurgitation being mild-moderate or moderate-severe. Using our system, we measured EROA values from all patients in both groups. We determined that the mean EROA value for the mild-moderate regurgitation patient group was $0.24 \pm 0.07 \mathrm{~cm}^{2}$ and the mean EROA value for the patient group with moderate-severe regurgitation was $0.43 \pm 0.13 \mathrm{~cm}^{2}$. These measurements were statistically distinguishable $(p<0.001)$, which indicated that our system can be used to distinguish these two patient populations.

\section{Discussion}

We have presented a system and workflow for fast acquisition and quantification of PISA, leading to accurate calculations of EROA and regurgitation volume. Our system utilizes 3D B-mode and Doppler flow volumetric echocardiography sequence data. To our knowledge, this is the first time that the complex 3D PISA diagnostic evaluation workflow was addressed by an efficient computational sys- 
tem. Extensive in vitro experiments establish that our system accurately measures surface area, EROA and regurgitant flow, especially as compared to the calculations obtained from the conventional spherical approximation. Further in vivo experiments with a patient population demonstrate that our system effectively measures EROA when cross-validated with a manual vena contracta measurement and that our system makes it possible to accurately distinguish patients with mild-moderate regurgitant flow from patients with moderate-severe regurgitant flow. In future work, the interactive specification of the valve and coaptation points may be replaced by an automatic localization.

\section{References}

1. Buck, T., Plicht, B., Hunold, P., Mucci, R., Erbel, R., Levine, R.: Broad-beam spectral Doppler sonification of the vena contracta using matrix-array technologya new solution for semi-automated quantification of mitral regurgitant flow volume and orifice area. J. Am. Coll. Cardiol. 45, 770-779 (2005)

2. DeGroff, C., Shandas, R., Valdes-Cruz, L.: Analysis of the effect of flow rate on the Doppler continuity equation for stenotic orifice area calculations: a numerical study. Circ. 97(16) (1998)

3. Enriquez-Sarano, M.: Timing of mitral valve surgery. Heart, 79-85 (2002)

4. Grady, L.: Random walks for image segmentation. IEEE Trans. on Pattern Analysis and Machine Intelligence 28(11), 1768-1783 (2006)

5. Lang, R., Mor-Avi, V., Sugeng, L., Nieman, P., Sahn, D.: Three-dimensional echocardiography: The benefits of the additional dimension. J. Am. Coll. Cardiol. 48, 20532669 (2006)

6. Little, S., Igo, S., McCulloch, M., Hartley, C., Nosé, Y., Zoghbi, W.: Three-dimensional ultrasound imaging model of mitral valve regurgitation: design and evaluation. Ultrasound in Med. Biol. 34(4), 647-654 (2008)

7. Matsumura, Y., Fukuda, S., Tran, H., Greenberg, N., Agler, D., Wada, N., Toyono, M., Thomas, J.: Geometry of the proximal isovelocity surface area in mitral regurgitation by 3-dimensional color Doppler echocardiography: difference between functional mitral regurgitation and prolapse regurgitation. AHJ, 155-231 (2008)

8. Pirat, B., Little, S., Igo, S., McCulloch, M., Nosé, Y., Hartley, C., Zoghbi, W.: Direct measurement of proximal isovelocity surface area by real-time threedimensional color Doppler for quantitation of aortic regurgitant volume: an in vitro validation. J. Am. Soc. Echo. 22(3), 306-313 (2009)

9. Schneider, R., Perrin, D., Vasilyev, N., Marx, G., del Nido, P., Howe, R.: Mitral annulus segmentation from 3D ultrasound using graph cuts. IEEE TMI 29(9), 1676-1687 (2010)

10. Singaraju, D., Grady, L., Vidal, R.: Interactive image segmentation of quadratic energies on directed graphs. In: Proc. of CVPR (June 2008)

11. Yosefy, C., Levine, R., Solis, J., Vaturi, M., Handschumacher, M., Hung, J.: Proximal flow convergence region as assessed by real-time 3-dimensional echocardiography: challenging the hemispheric assumption. J. Am. Soc. Echo. (2007)

12. Zoghbi, W., Enriquez-Sarano, M., Foster, E., Grayburn, P., Kraft, C., Levine, R., Nihoyannopoulos, P., Otto, C., Quinones, M., Rakowski, H.: Recommendations for evaluation of the severity of native valvular regurgitation with two-dimensional and Doppler echocardiography. J. Am. Soc. Echo. 16, 777-802 (2003) 